\title{
Enhancement of reading ability using self- controlled gaze interface in a digital medium for children with neuro-oculomotor challenges
}

\author{
Louisa M. S. Wong \\ HK Red Cross Princess Alexandra School \\ 8 Rehab Path, Kwun Tong, KIn., HKG \\ 14017654g@connect.polyu.hk
}

\author{
Carly S. Y. Lam \\ School of Optometry \\ The Hong Kong Polytechnic University \\ carly.lam@polyu.edu.hk
}

\begin{abstract}
There is ample research on the effectiveness of oculomotor training (OMT) to regain reading efficiency in Alphabetic language systems for many years, not only for learning disabilities but also applicable to patients with acquired brain injury. In addition, the advancement of eye tracking technology and computation model of reading, allowing researchers to enter new horizons in studying reading-related oculomotor (OM) skills. Yet there is a lack of studies in using OMT to enhance reading skills in Chinese for children with neuromuscular disorders (NMD) associated with congenital OM challenges. Our hypothesis is that by using self-directed gaze-interface to access a curriculum-based digital reading platform, it enhances the OM control of children with NMD during reading in Chinese; which in terms of accurate fixation to the area of interest (AOI), decreasing saccadic dysmetria, and better result in orthographic recognition in comparison with children without OMT. It is a cross-centres prospective Cohort study with quasi-experimental design. 21 students with NMD, aged 6 to 8 years with oculomotor challenges completed 8-weeks of interventions then followed by 4 months of repeated measures. Repeated measures showed improved and sustainable effect in treatment group than control group, such as accurate saccade and better revision result. Data analysis of between-group and within-group difference, and factor analysis is still in progress.
\end{abstract}

reading-related oculomotor skills, digital reading, Chinese, eye tracking, fixation, saccade, $\mathrm{HCl}$, neuromuscular disorders

\section{INTRODUCTION}

Neuromuscular disorder (NMD) is an umbrella term for movement disorders originating in central nervous systems resulting in motor and postural dysfunctions [1, 2], including reading-related $\mathrm{OM}$ anomalies [3-6]. A number of visual problems had been identified with NMD (about 70-90\% with stabismus, nearly $100 \%$ showed some oculomotor anomalies, etc.) with the core problem being reduced reading performance [6-8]. When the core problem of reading-related OM skills in children with NMD is not addressed properly, it leads to a diminished learning capability. Helping these children to acquire Chinese (mother-tongue) reading-related OM skills may have significant beneficial effects to both educational and rehabilitation development in a Chinese language environment.

Previous studies of neuroplasticity of OM explained that the 12 extraocular muscles (EOM) moved in groups of agonist and antagonist to maintain the eyes in same direction for good vision [8, 9].

Therefore, eye movements are translated into absolute motor output [10], it can be measured in range of motion (visual angle and smooth saccade), muscle grading (fixation dwell for image drift), and muscles coordination (saccadic sequences). It is coherent with the theory of neuroplasticity and motor learning principles [11, $12]$, by repeated practice to gain automaticity with adjustable subsequent of movements [13].

Our study will use Tobii PCEye in reading tasks through a web-based digital reading programme (Eye C.U.R.E. 1) - a computational model of curriculum-based reading which is activated by a user's eye movement via corneal reflection. The digital reading process incorporated with the training media of guided and graded exercises for EOM.

\section{LITERATURE REVIEW}

\subsection{Reading-related visual skills}

Vision related reading problems can be divided into two broad visual systems: a low order of "visual efficiency" and the high order of "visual information processing" [3]. Visual efficiency refers to basic physiological processes of visual acuity, accommodation $^{2}$, vergence ${ }^{3}$ and $\mathrm{OM}$ control ${ }^{4}-$

\footnotetext{
${ }^{1}$ Eye C.U.R.E. stand for Eye Can Understand and Read Easily; it is a computational model of reading platform, originally designed by the author, programming and technical supported by the Panel Resources Ltd and school IT team.

${ }^{2}$ Accommodation: the ability of an eye to focus clearly on objects at various distance. [3]

${ }^{3}$ Vergence is the simultaneously eyes movement in opposite
} 
Warren [15] defined this level of visual function as the foundation of visual-cognition hierarchy. The higher order of visual information processing not only refers to accuracy of these physiological processes, but also to the non-motor aspects of the visual perception, cognition, language, and attention integration.

From previous neurophysiological studies, visual efficiency is the pre-requisite to reading $[3,16]$. Optometrists found that babies practice eye muscles for visual efficiency frequently before 4 years of age; by age 5, visual functions such as visual acuity, accommodation and vergence become mature [17, 18]. EOM, which brings about binocular single vision, fixation, visual tracking, also matures at around 5 to 6 years old together with other visual functions. Normal eye movements are pre-requisites for stable and clear vision at all distances (normal accommodation), and normal binocular fusion is a pre-requisite for single binocular vision during reading. Researchers who studied vision and visual processing, found that the OM function is essential to reading [19-23].

Ciuffreda [24] confirmed that the motor training of eye muscles have benefits and contribute to reading in terms of gaining cognition and attention due to saccade efference copies ${ }^{5}$ of motor commands [25], known as corollary discharge and incorporated into forward models of saccade, that is following the sensory consequences/ proprioception of EOM, to initate a second saccade after fixation [26, 27]. Such findings about the adapatability of EOM were in line with the neuroplasticity and motor training principles $[8,10]$. Accordingly, OMT can be performed for smooth saccade during reading, eye movements being gradated movements of EOM $[6,10]$.

\subsection{Chinese language reading}

Some studies suggested the eye movement of reading Chinese is similar to English [28-30], but others commented that the structure of Chinese characters and words modulated the place of fixation and saccadic length and were different from the reading of English [31-35]. Some researchers argued that the absence of visual word segmentation is the key feature which differentiates Chinese [36, 37] from the parallel visual-word processing of reading English [32, 36]. Bai [31] reported that Chinese words are the units of primary importance in visual tracking. Yen [32] noted that Chinese sentences lack cues for word boundaries, the eye movements seem substantially

direction (Leigh \& Zee, 2006), away from nasal field.

${ }^{4}$ Oculomotor control, eye movements and fixation/ saccade are inter-changeable in this proposal.

${ }^{5}$ Efferent copying, knows as Corollary discharge; is a copy of a motor command that is sent to the muscles to produce a movement different from reading English. Siok [38] also suggested that reading Chinese is based on repetition memorization of the pronunciation of Chinese characters instead of phonological awareness when reading in English.

Meta-analysis recognized that visual skills have medium effect $(r=0.32)$ when associated with Chinese reading acquisition [39], but most research were only done on the effectiveness of OMT to regain reading efficiency in Alphabetic language systems $[40,41]$ for learning disabilities $[42,43]$ and patients with acquired brain injury [16, 24, 44]. There is a lack of studies on using OMT to enhance saccade and fixation in reading of Chinese for children with NMD associated with OM anomalies.

\subsection{Ocular Motor Training and its benefits}

Children with NMD not only have difficulty accessing reading materials because of diminished motor output, but also have deficits in OM skill for reading and hence slow progress in academic performance; leading to fewer people achieving higher levels of learning. Therefore early intervention in primary level to enhance children's OM control primarily from performance components of visual search (saccade) and attention (fixation) to functional reading is very important.

From the motor learning prespective, reading is considered as a visual skill of saccade and fixation, it provides a bottom-up approach for intervention of reading acquisition, by using repeated OMT. Regarding advance eye technology and humancomputer interaction, new research using gazeinterface to access digital Chinese reading platforms help children with reading-related OM anomalies; and also help clinicians to establish evidence-based protocols for goal-specific interventions.

\section{THE APPLICATION IDEA OF 'EYECURE'}

Therapists in special schools wish to facilitate silent reading for a group of children with multiple challenges in speech, visual and motors, to enable their independent learning as well as to maximize their learning capacity. EyeCURE, a web-based gaze-accessible and self-contained reading platform in Chinese is used for this purpose. The system asks for EOM synergistics in one group for saccade from one target to another, even though each eye has three degrees of freedoms (DOF) but the brain always resolve it by Listing's Law, i.e. decide on a special simple reference/area of interest (AOI) [10]. The development of this guided reading platform aims at facilitating beginner readers/children with $\mathrm{OM}$ anomalies to adapt where to look, what to attend to, and how to time fixate 
information with accurate saccade length and landing. The gaze can be directed to only one location at a time and information central to success must be selected from a spatically complex environment [45].

Basically, the programme of EyeCURE facilitates the most fundamental version saccade from left to right only. So subjects are forced to practise the saccade for reading from left to right. Repeated motor exercises of EOM are adapted to corollary discharge in primate vision [27], i.e. from intentional/voluntary muscle output translated into automatic/reflexive movement through repetitive practice.

\subsection{The principle of EyeCURE}

For best vision of reading, images must be brought to the fovea by accurate fixation to the retina and held there with image drift less than about $5 \% / \mathrm{sec}$ [8]. Typical fixation frequency for reading is $<3 \mathrm{~Hz}^{6}$. As neurophysiology and eye technology advances, the motor mechanism of fixation and saccade are measurable and gradable by computer programming. The metric of fixation and saccade in terms of reading span in EyeCURE can be programmed through $1-3 \mathrm{~Hz}$ by manipulating the gaze-interface pointer speed and the rate of readaloud function. To facilitate a successful image drift by good fixation, therapists can calculate pixels of Chinese characters and phrases lengths to ensure that the visual angle is limited to $5 \% / \mathrm{sec}$.

In EyeCURE, curriculum-based reading in Chinese will be presented to the subjects. The subjects shall use their gaze movement (activated by the Tobii PCEye) to browse the Chinese characters, phrases and passages. The pointer movement is gaze-activated by smooth saccade from left to right, and the read aloud text-to-speech engine will be activated by the mouse-over action which is interfaced by fixation dwell spontaneously. (Fig.1)

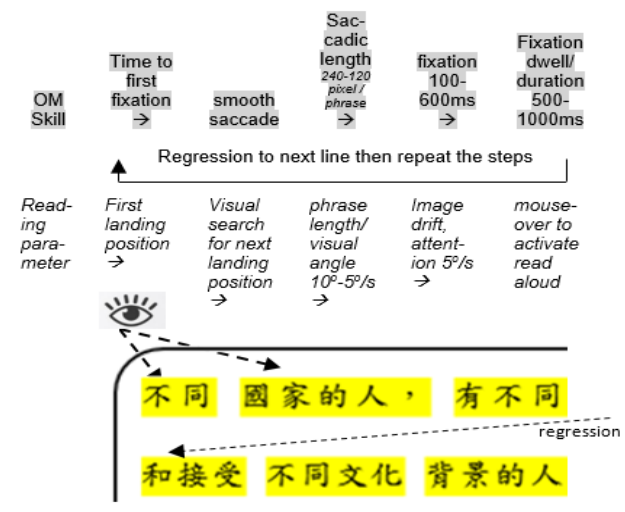

Figure 1: logistic of gaze-interface OMT via EyeCURE

\footnotetext{
${ }^{6}$ The Eye Tracking Metric in Tobii Studio

http://class.classmatandread.net/Eye/eye metrics.pdf
}

The treatment principle is guided and graded OMT, to alter the number of phrase/fixation is equivalent to manipulating the flexibility of eye muscles; the reading angle, visual span as well as the saccade length can be graded in terms of phrase length.

Tobii PCEye can be set to vary pointer speed, faster or slower, calibrate from slowest-0 to fastest100 in $10 \%$ increments. So, it can guide the user to hold the gaze to get a relatively steady foveal image; it is the neurophysiology of the eye that clear vision images are formed by holding a fairly steady fixation on the foveal at about $5 \% / \mathrm{sec}$ [8]. The gradation variables of EyeCURE for OMT are:

- pointer speed = visual search / smooth saccade;

- $\quad$ range of motion $=$ visual angle $/$ saccade length;

- $\quad$ mouse-over time = fixation duration;

- $\quad$ reading rate $=$ number of fixation; and

- reading accuracy in terms of comprehension in multiple choice format which is activated and selected by vertical eye movement only (see appendix).

\subsection{Intervention of guided and graded OMT}

EyeCURE is an open system that allow teachers and therapists to input different levels of reading materials with respect to the curriculum of Chinese Language of the primary level.

The number of choices of passage can be altered by teachers or therapists with respect to the grade levels. The details of setup and illustration of the eplatform is presented in the appendix.

In this study, student will receive EOM training of saccade and fixation by means of the gaze-pointer interface of Tobii PCEye. It is aimed at translating the reading behaviour to absolute OM control according to the principles of motor learning and neuroplasticity for therapeutic and research purposes.

In the EyeCURE programme, we limit the mouse over to three functions only:

(1) pointer horizontal move is activated by saccade, aimed at guided version reading from left to right then regression from right to left for next line;

(2) mouse over for fixation dwell to activate readaloud; dwell time from $0.5 \mathrm{sec}$ to $1 \mathrm{sec} /$ selection; and

(3) pointer vertical move is default as 'enter' for selection.

Either the human-computer interaction between user and Tobii, or human-system interaction between user and EyeCURE, can be adjusted and calibrated with respect to a user's condition to seek the optimum performance. Tobii allows multiusers 
and can save different profiles for personalised setup.

\section{METHODOLOGY}

4.1 Experimental design: prospective Cohort study with quasi-experimental design

\section{$\underline{\text { Sample size calculation }}$}

Given that no previous studies have considered the effectiveness of self-directed OMT in reading of Chinese, the estimated improvement in readingrelated OM control in 8 weeks with medium effect size about 0.3 , power to $90 \%$ at $p<0.05$, the total sample size for 4 repeated measurements on the same subjects is 18 . Finally, there were 21 subjects who completed 4 repeated measures, from Nov. 2016 to April 2017.

\subsection{Subject Characteristics and Inclusion criteria}

Because of the very specific area of study, subjects will be recruited from special schools for children with physical disabilities, which is a known population but with small numbers. Potential candidates suffer from NMD associated with OM anomalies, study in primary school of normal intelligence and with native language in Chinese and Cantonese; grade 1 to 3 , about age 6 to 8 ; other inclusion criteria are as follows:

1. because of pure OMT, subjects with dysarthria may also be recruited;

2. students with normal to mild visual impairment (best corrected visual acuity is better than 20/60);

3. both eyes without visual loss as Tobii PCeye and tracker X3-120 (assessment tool) seek binocular vision for calibration and assessment.

4.3 Exclusion criteria: (1) students with mild to moderate intellectual disability; (2) students unable to read Chinese or unable to understand spoken Cantonese; (3) student with moderate to severe visual impairment; (4) students with ADHD, ASD or with behaviour challenging and attention problems, and/or with epileptic seizure elicited by computer flickering lights.

\subsection{Data processing and analysis}

Demographic and ocular data will be summarized with descriptive statistics: mean, median, range, standard deviation, $90 \% \mathrm{Cl}$, as appropriate. All statistical analyses of this research of parametric and quantitative data will be performed using SPSS. Set the $p<0.05$ for one-tail analysis (statistical significant to reject $H_{0}$ ). A paired t-test will be conducted to compare the means of with-in subject performance of Tobii measurement on fixation and saccade pre and post training on the horizontal OM control. Repeated measures analysis of variance (ANOVA) will apply to measure the change over time, to compare between group differences about pre-/ post-/ sustained- measures of the treatment group and control group. Regression analysis will be applied to estimate the relationships among variables.

Repeated measures showed improved and sustainable effect in treatment group than control group; in the parameters such as accurate smooth saccade, fixation count, shorter total visit duration and better revision result in orthographic recognition of Chinese characters and words phrases.

Data processing in progress. Further analysis will investigate between-group and within-group difference, multiple regression to reveal relationships among human factors and system factors.

\section{THE IMPACT AND CONTRIBUTIONS TO THE FIELD}

This project is a transdisciplinary effort to tackle the reading-related OM problem in children with NMD. It demonstrates the synergy among occupational therapists, optometrists, teachers, and IT professions to benefit children with deprivation from formal learning media. Significantly, this is a novel study about gaze-access to a digital Chinesereading platform for children with NMD. This target group are being excluded from ordinary educational media, not only due to inaccessibility to physical books or computers, but also dysarthria; resulting in poor academic performance. The philosophy of research design is anchored in motor learning theory and neuroplasticity, which has had evidence-based practice. Therefore, this research will evaluate gaze-directed OMT via humancomputer-interaction and its relationship to reading in Chinese. The test results may give insights to rehabilitation professionals and educators to develop an early intervention protocol of readingrelated OMT and possibly further generalize to other groups of subjects with reading-related OM challenges, for examples, autistic spectrum disorder, dyslexia, degenerative disorders, etc. Findings from this study will shed light on the possible enhancement of research about readingrelated OM skills, and enabling people with OM challenges to achieve better academic qualifications and thus enhance their quality of life and career prospects!

Conflict of Interest: The authors declare that this research was conducted in the absence of any 
commercial or financial relationships that could be construed as a potential conflict of interest.

Acknowledgement: The study was supported by $\mathrm{ZOH1}$ from The Hong Kong Polytechnic University; and special thanks to the Red Cross Princess Alexandra School, JFK Centre, Pui Oi School, and the Tronda Electronics Ltd., without their support this project would not been possible.

\section{REFERENCES}

1. Bobath, K. (1980). A neurophysiological basis for the treatment of cerebral palsy. 2nd ed. Clinics in developmental medicine no 75. London: Spastics International Medical Publications ;Heinemann Medical etc.

2. Mayston, M., Damiano, D. and Scrutton, D. (2004). Management of the motor disorders of children with cerebral palsy. 2nd ed. Clinics in developmental medicine. London: Mac Keith Press. x, 204 p.

3. Garzia, R. P., Borsting, Eric J., Nicholson, Steven B., Press, Leonard J., Scheiman, Mitchell M., Solan, Harold A. (Ed.) (2008). Optometric clinical practice guideline care of the patient with learning related vision problems. Reference Guide for Clinicians. http://www.aoa.org/documents/optometrists /CPG-20.pdf

4. Sellier, E., Uldall, P., Calado, E., Sigurdardottir, S., Torrioli, M. G., Platt, M. J., \& Cans, C. (2012). Epilepsy and cerebral palsy: Characteristics and trends in children born in 1976-1998. European Journal of Paediatric Neurology, 16(1), 4855.

doi:http://dx.doi.org/10.1016/i.ejpn.2011.10. $\underline{003}$

5. $\overline{\mathrm{Dan}}$, B., Mayston, M., Paneth, N., \& Rosenbloom, L. (2014). Cerebral palsy : science and clinical practice. London: Mac Keith Press.

6. Whittaker, S., Scheiman, M., \& SokolMcKay, D. (2015). Low Vision Rehabilitation, A Practical Guide for Occupational Therapists (2nd ed.). USA: SLACK Inc.

7. Scheiman, M., \& Rouse, M. W. (2006). Optometric management of learningrelated vision problems (2nd ed.). St. Louis, Mo: St. Louis, Mo. : Mosby-Elsevier.

8. Leigh, R. J., \& Zee, D. S. (2006). The neurology of eye movements (4th ed.). New York, N.Y: New York, N.Y. : Oxford University Press.

9. Riordan-Eva, P., Cunningham, E. T., Vaughan, D., \& Asbury, T. (2011). Vaughan \& Asbury's general ophthalmology General ophthalmology (18th ed. / edited by Paul Riordan-Eva, Emmett T. Cunningham Jr. ed.). New York: New York : McGraw-Hill Medical.

10. Werner, J. S., Chalupa, L. M., Burns, M. E., Geng, J. J., Goldman, M. S., Handa, J., . . . ebrary, I. (2014). The new visual neurosciences. Cambridge, Massachusetts: The MIT Press.

11. Massone, L. (1994). A neural-network system for control of eye movements: basic mechanisms. Biological Cybernetics, 71(4), 293-305. doi: 10.1007/BF00239617

12. Latash, M. L., ebrary, I., \& ScienceDirect. (2012). Fundamentals of motor control. London: Academic Press.

13. Zwicker, J. G., \& Harris, S. R. (2009). A Reflection on Motor Learning Theory in Pediatric Occupational Therapy Practice. Canadian Journal of Occupational Therapy, 76(1), 29-37.

14. Fazzi, E., Signorini, S. G., La Piana, R., Bertone, C., Misefari, W., Galli, J., Bianchi, P. E. (2012). Neuro-Ophthalmological Disorders in Cerebral Palsy: Ophthalmological, Oculomotor, and Visual Aspects. Developmental Medicine \& Child Neurology, 54(8), 730-736.

doi: 10.1111/j.1469-8749.2012.04324.x

15. Warren, M. (1993). A hierarchical model for evaluation and treatment of visual perceptual dysfunction in adult acquired brain injury, Part 1. The American journal of occupational therapy : official publication of the American Occupational Therapy Association, 47(1), 42.

16. Thiagarajan, P., Ciuffreda, K. J., CapoAponte, J. E., Ludlam, D. P., \& Kapoor, N. (2014). Oculomotor neurorehabilitation for reading in mild traumatic brain injury (mTBI): an integrative approach. NeuroRehabilitation, 34(1), 129.

17. Hyvärinen, L. (1988). Vision in children, normal and abnormal. Meaford, Ont: Canadian Deaf-Blind \& Rubella Assn.

18. Tasker, R. C., McClure, R. J., Acerini, C. L., \& ebrary, I. (2013). Oxford handbook of paediatrics Paediatrics (2nd ed.). Oxford: Oxford: Oxford University Press.

19. Allen, P. M., Bruce JW Evans and Arnold J Wilkins. (2009). Vision and Reading Difficulties. London: Ten Apls Creative.

20. Feng, G., Miller, K., Shu, H., \& Zhang, H. (2009). Orthography and the Development of Reading Processes: An Eye-Movement Study of Chinese and English. Child Development, 80(3), 720-735.

21. Jacobson, L., Ygge, J., Flodmark, O., \& Ek, U. (2002). Visual and perceptual characteristics, ocular motility and 
strabismus in children with periventricular leukomalacia. Strabismus, 10(2), 179.

22. Pennefather, P. M., \& Tin, W. (2000). Ocular abnormalities associated with cerebral palsy after preterm birth. Eye, 14(1), 78-81. doi: 10.1038/eye.2000.17

23. Vidyasagar, T. R., \& Pammer, K. (2010). Dyslexia: a deficit in visuo-spatial attention, not in phonological processing. Trends in Cognitive Sciences, 14(2), 57-63. doi: 10.1016/j.tics.2009.12.003

24. Ciuffreda, K. J., Han, Y., Kapoor, N., \& Ficarra, A. P. (2006). Oculomotor rehabilitation for reading in acquired brain injury. NeuroRehabilitation, 21(1), 9.

25. Peterburs, J., Koch, B., Schwarz, M., Hoffmann, K. p., Daum, I., \& Bellebaum, C. (2013). Cortical processing of saccaderelated efference copy signals in patients with cerebellar lesion. European Journal of Neuroscience, 37(5), 804-815. doi: 10.1111/ejn.12081

26. Cole, M. J., Gwizdka, J., Liu, C., Belkin, N. J., \& Zhang, X. (2013). Inferring user knowledge level from eye movement patterns. Information Processing and Management, 49(5), 1075-1091.

doi: 10.1016/j.ipm.2012.08.004

27. Wurtz, R. (2013). Corollary discharge in primate vision. Scholarpedia, 8(10), 12335. doi: 10.4249/scholarpedia.12335

28. Cui, L., Yan, G., Bai, X., Hyönä, J., Wang, S., \& Liversedge, S. P. (2013). Processing of compound-word characters in reading Chinese: An eye-movement-contingent display change study. The Quarterly Journal of Experimental Psychology, 66(3), 527-547.

doi: 10.1080/17470218.2012.667423

29. Rayner, K., Li, X., \& Pollatsek, A. (2007). Extending the E-Z Reader Model of Eye Movement Control to Chinese Readers. Cognitive Science, 31(6), 1021-1033.

doi: 10.1080/03640210701703824

30. McBride-Chang, C., \& Kail, R. V. (2002). Cross-Cultural Similarities in the Predictors of Reading Acquisition. Child Development, 73(5), 1392-1407.

31. Bai, X., Yan, G., Liversedge, S. P., Zang, C., \& Rayner, K. (2008). Reading Spaced and Unspaced Chinese Text: Evidence From Eye Movements. Journal of Experimental Psychology: Human Perception and Performance, 34(5), 12771287. doi: 10.1037/0096-1523.34.5.1277

32. Yen, M.-H., Radach, R., Tzeng, O. J. L., Hung, D. L., \& Tsai, J.-L. (2009). Early parafoveal processing in reading Chinese sentences. Acta Psychologica, 131(1), 2433. doi: 10.1016/j.actpsy.2009.02.005
33. Li, X., Liu, P., \& Rayner, K. (2011). Eye movement guidance in Chinese reading: Is there a preferred viewing location? Vision Research, 51(10), 1146-1156. doi: 10.1016/j.visres.2011.03.004

34. Wei, W., Li, X., \& Pollatsek, A. (2013). Word properties of a fixated region affect outgoing saccade length in Chinese reading.(Report). Vision Research, 80, 1.

35. Ma, G., \& Li, X. (2015). How character complexity modulates eye movement control in Chinese reading. $A n$ Interdisciplinary Journal, 28(6), 747-761. doi: 10.1007/s11145-015-9548-1

36. Radach, R., \& Kennedy, A. (2013). Eye movements in reading: Some theoretical context. The Quarterly Journal of Experimental Psychology, 66(3), 429-452. doi: 10.1080/17470218.2012.750676

37. 白学军, 李馨, 回国利, \& Guoli, B. A. I. X. L. X. Y. (2015). 汉语阅读眼动控制：20 年研究 的总结 - Eye Movement Control in Chinese Reading: A Summary over the Past 20 Years of Research. 心理发展与教育, 31(01), 85-91.

38. Siok, W. T., Spinks, J. A., Jin, Z., \& Tan, L. H. (2009). Developmental dyslexia is characterized by the co-existence of visuospatial and phonological disorders in Chinese children. Current Biology, 19(19), R890-R892. doi: http://dx.doi.org/10.1016/i.cub.2009.08.014

39. Yang, L.-Y., Guo, J.-P., Richman, L., Schmidt, F., Gerken, K., \& Ding, Y. (2013). Visual Skills and Chinese Reading Acquisition: A Meta-analysis of Correlation Evidence. Educational Psychology Review.

40. Gaertner, C., Bucci, M. P., Ajrezo, L., \& Wiener-Vacher, S. (2013). Binocular coordination of saccades during reading in children with clinically assessed poor vergence capabilities. Vision Research, 87, 22-29. doi: 10.1016/j.visres.2013.05.006

41. Leong, D. F., Master, C. L., Messner, L. V., Pang, Y., Smith, C., \& Starling, A. J. (2014). The Effect of Saccadic Training on Early Reading Fluency. Clinical Pediatrics, 53(9), 858-864. doi: 10.1177/0009922814532520

42. Sigler, G. (1994). The effect of Vision Therapy on reading rate: a pilot study. Journal of Behavioral Optometry, 5(4), 5.

43. Marrs, H., \& Patrick, C. (2002). A Return to Eye-Movement Training? An Evaluation of the Reading Plus Program. Reading Psychology, 23(4), 297-322.

44. Han, Y., Ciuffreda, K. J., \& Kapoor, N. (2004). Reading-related oculomotor testing and training protocols for acquired brain 
injury in humans. Brain Research Protocols, 14(1), 1-12.

doi: 10.1016/j.brainresprot.2004.06.002

45. Vickers, J. N. (2011). Mind over muscle: the role of gaze control, spatial cognition, and the quiet eye in motor expertise (Vol. 12, pp. 219). 